\title{
ON COINCIDENCE POINT THEOREM FOR NEW CONTRACTIVE CONDITION WITH APPLICATION
}

\author{
Bhavana Deshpande and Amrish Handa
}

\begin{abstract}
We establish a coincidence point theorem for $g$-non-decreasing mappings satisfying a new contractive condition on partially ordered metric spaces. With the help of the obtained result, we show the formation of the coupled coincidence point theorem for generalized compatible pair of mappings $F, G: X^{2} \rightarrow X$. Furthermore, an example and an application to integral equations are also given to show the usefulness of the obtained results. Our results generalize, modify, improve and sharpen several well-known results.

Keywords: Coincidence point; Coupled coincidence point; Contractive condition; Partially ordered metric space; O-compatibility; Generalized compatibility; $g$-non-decreasing mapping; Mixed monotone mapping; Commuting mapping
\end{abstract}

\section{Introduction and Preliminaries}

In the sequel, we denote by $X$ a non-empty set and $\preceq$ will represent a partial order on $X$. Given $n \in \mathbb{N}$ with $n \geq 2$, let $X^{n}$ be the nth Cartesian product $X \times X \times$ $\ldots \times X$ (n times). For simplicity, if $x \in X$, we denote $g(x)$ by $g x$.

The idea of the coupled fixed point was initiated by Guo and Lakshmikantham [10] in 1987. In [10], Guo and Lakshmikantham introduced the following notion of coupled fixed point for single-valued mappings:

Definition 1.1. Let $F: X^{2} \rightarrow X$ be a given mapping. An element $(x, y) \in X^{2}$ is called a coupled fixed point of $F$ if

$$
F(x, y)=x \text { and } F(y, x)=y \text {. }
$$

Following this paper, Gnana-Bhaskar and Lakshmikantham [3] introduced the notion of mixed monotone property for $F: X^{2} \rightarrow X$ (where $X$ is an ordered

Received December 24, 2015; accepted April 03, 2016

2010 Mathematics Subject Classification. Primary 47H10; Secondary 54H25 
metric space) and utilized the same to prove some theorems on the existence and uniqueness of coupled fixed points.

In [3], Gnana-Bhaskar and Lakshmikantham introduced the following:

Definition 1.2. Let $(X, \preceq)$ be a partially ordered set. Suppose $F: X^{2} \rightarrow X$ is a given mapping. We say that $F$ has the mixed monotone property if for all $x, y \in X$, we have

$$
x_{1}, x_{2} \in X, x_{1} \preceq x_{2} \Longrightarrow F\left(x_{1}, y\right) \preceq F\left(x_{2}, y\right),
$$

and

$$
y_{1}, y_{2} \in X, y_{1} \preceq y_{2} \Longrightarrow F\left(x, y_{1}\right) \succeq F\left(x, y_{2}\right) .
$$

In 2009, Lakshmikantham and Ciric [16] generalized these results for nonlinear contractive mappings by introducing the notions of coupled coincidence point and mixed g-monotone property.

In [16], Lakshmikantham and Ciric introduced the following concepts:

Definition 1.3. Let $F: X^{2} \rightarrow X$ and $g: X \rightarrow X$ be given mappings. An element $(x, y) \in X^{2}$ is called a coupled coincidence point of the mappings $F$ and $g$ if

$$
F(x, y)=g x \text { and } F(y, x)=g y .
$$

Definition 1.4. Let $F: X^{2} \rightarrow X$ and $g: X \rightarrow X$ be given mappings. An element $(x, y) \in X^{2}$ is called a common coupled fixed point of the mappings $F$ and $g$ if

$$
x=F(x, y)=g x \text { and } y=F(y, x)=g y .
$$

Definition 1.5. The mappings $F: X^{2} \rightarrow X$ and $g: X \rightarrow X$ are said to be commutative if

$$
g F(x, y)=F(g x, g y), \text { for all }(x, y) \in X^{2} .
$$

Definition 1.6. Let $(X, \preceq)$ be a partially ordered set. Suppose $F: X^{2} \rightarrow X$ and $g: X \rightarrow X$ are given mappings. We say that $F$ has the mixed $g$-monotone property if for all $x, y \in X$, we have

$$
x_{1}, x_{2} \in X, g x_{1} \preceq g x_{2} \Longrightarrow F\left(x_{1}, y\right) \preceq F\left(x_{2}, y\right),
$$

and

$$
y_{1}, y_{2} \in X, g y_{1} \preceq g y_{2} \Longrightarrow F\left(x, y_{1}\right) \succeq F\left(x, y_{2}\right) .
$$

If $g$ is the identity mapping on $X$, then $F$ satisfies the mixed monotone property.

Subsequently, Choudhury and Kundu [4] introduced the notion of compatibility and used this notion to improve the results of Lakshmikantham and Ciric [16]. Several authors established coupled fixed/coincidence point theorems by using this notion. 
Definition 1.7. [4]. The mappings $F: X^{2} \rightarrow X$ and $g: X \rightarrow X$ are said to be compatible if

$$
\begin{aligned}
& \lim _{n \rightarrow \infty} d\left(g F\left(x_{n}, y_{n}\right), F\left(g x_{n}, g y_{n}\right)\right)=0, \\
& \lim _{n \rightarrow \infty} d\left(g F\left(y_{n}, x_{n}\right), F\left(g y_{n}, g x_{n}\right)\right)=0,
\end{aligned}
$$

whenever $\left\{x_{n}\right\}$ and $\left\{y_{n}\right\}$ are sequences in $X$ such that

$$
\begin{aligned}
& \lim _{n \rightarrow \infty} F\left(x_{n}, y_{n}\right)=\lim _{n \rightarrow \infty} g x_{n}=x, \\
& \lim _{n \rightarrow \infty} F\left(y_{n}, x_{n}\right)=\lim _{n \rightarrow \infty} g y_{n}=y, \text { for some } x, y \in X .
\end{aligned}
$$

A great deal of these studies investigate contractions on partially ordered metric spaces because of their applicability to initial value problems defined by differential or integral equations.

Hussain et al. [12] introduced the notion of generalized compatibility of a pair $\{F, G\}$, of mappings $F, G: X \times X \rightarrow X$, then the authors employed this notion to obtained coupled coincidence point results for such a pair of mappings involving $(\varphi, \psi)$-contractive condition without mixed $G$-monotone property of $F$.

In [12], Hussain et al. introduced the following:

Definition 1.8. Suppose that $F, G: X^{2} \rightarrow X$ are two mappings. $F$ is said to be $G$-increasing with respect to $\preceq$ if for all $x, y, u, v \in X$, with $G(x, y) \preceq G(u, v)$ we have $F(x, y) \preceq F(u, v)$.

Definition 1.9. Let $F, G: X^{2} \rightarrow X$ be two mappings. We say that the pair $\{F$, $G\}$ is commuting if

$$
F(G(x, y), G(y, x))=G(F(x, y), F(y, x)), \text { for all } x, y \in X .
$$

Definition 1.10. Suppose that $F, G: X^{2} \rightarrow X$ are two mappings. An element $(x, y) \in X^{2}$ is called a coupled coincidence point of mappings $F$ and $G$ if

$$
F(x, y)=G(x, y) \text { and } F(y, x)=G(y, x) .
$$

Definition 1.11. Let $(X, \preceq)$ be a partially ordered set, $F: X^{2} \rightarrow X$ and $g: X \rightarrow$ $X$ are two mappings. We say that $F$ is $g$-increasing with respect to $\preceq$ if for any $x$, $y \in X$,

$$
g x_{1} \preceq g x_{2} \text { implies } F\left(x_{1}, y\right) \preceq F\left(x_{2}, y\right),
$$

and

$$
g y_{1} \preceq g y_{2} \text { implies } F\left(x, y_{1}\right) \preceq F\left(x, y_{2}\right) \text {. }
$$


Definition 1.12. Let $(X, \preceq)$ be a partially ordered set, $F: X^{2} \rightarrow X$ be a mapping. We say that $F$ is increasing with respect to $\preceq$ if for any $x, y \in X$,

$$
\begin{aligned}
& x_{1} \preceq x_{2} \text { implies } F\left(x_{1}, y\right) \preceq F\left(x_{2}, y\right), \\
& y_{1} \preceq y_{2} \text { implies } F\left(x, y_{1}\right) \preceq F\left(x, y_{2}\right) .
\end{aligned}
$$

Definition 1.13. Let $F, G: X^{2} \rightarrow X$ are two mappings. We say that the pair $\{F, G\}$ is generalized compatible if

$$
\begin{aligned}
& \lim _{n \rightarrow \infty} d\left(F\left(G\left(x_{n}, y_{n}\right), G\left(y_{n}, x_{n}\right)\right), G\left(F\left(x_{n}, y_{n}\right), F\left(y_{n}, x_{n}\right)\right)\right)=0, \\
& \lim _{n \rightarrow \infty} d\left(F\left(G\left(y_{n}, x_{n}\right), G\left(x_{n}, y_{n}\right)\right), G\left(F\left(y_{n}, x_{n}\right), F\left(x_{n}, y_{n}\right)\right)\right)=0,
\end{aligned}
$$

whenever $\left(x_{n}\right)$ and $\left(y_{n}\right)$ are sequences in $X$ such that

$$
\begin{aligned}
\lim _{n \rightarrow \infty} G\left(x_{n}, y_{n}\right) & =\lim _{n \rightarrow \infty} F\left(x_{n}, y_{n}\right)=x, \\
\lim _{n \rightarrow \infty} G\left(y_{n}, x_{n}\right) & =\lim _{n \rightarrow \infty} F\left(y_{n}, x_{n}\right)=y, \text { for some } x, y \in X .
\end{aligned}
$$

Obviously, a commuting pair is generalized compatible but not conversely in general.

Erhan et al. [8], announced that the results established in Hussain et al. [12] can be easily derived from the coincidence point results in the literature.

In [8], Erhan et al. recalled the following basic definitions:

Definition 1.14. $[\mathbf{1}, \mathbf{9}]$. A coincidence point of two mappings $T, g: X \rightarrow X$ is a point $x \in X$ such that $T x=g x$.

Definition 1.15. [8]. A partially ordered metric space $(X, d, \preceq)$ is a metric space $(X, d)$ provided with a partial order $\preceq$.

Definition 1.16. $[3,12]$. A partially ordered metric space $(X, d, \preceq)$ is said to be non-decreasing-regular (respectively, non-increasing-regular) if for every sequence $\left\{x_{n}\right\} \subseteq X$ such that $\left\{x_{n}\right\} \rightarrow x$ and $x_{n} \preceq x_{n+1}$ (respectively, $x_{n} \succeq x_{n+1}$ ) for all $n \geq 0$, we have that $x_{n} \preceq x$ (respectively, $x_{n} \succeq x$ ) for all $n \geq 0$. $(X, d, \preceq$ ) is said to be regular if it is both non-decreasing-regular and non-increasing-regular.

Definition 1.17. [8]. Let $(X, \preceq)$ be a partially ordered set and let $T, g: X \rightarrow X$ be two mappings. We say that $T$ is $(g, \preceq)$-non-decreasing if $T x \preceq T y$ for all $x$, $y \in X$ such that $g x \preceq g y$. If $g$ is the identity mapping on $X$, we say that $T$ is $\preceq$-non-decreasing.

Remark 1.1. [8]. If $T$ is $(g, \preceq)$-non-decreasing and $g x=g y$, then $T x=T y$. It follows that

$$
g x=g y \Rightarrow\left\{\begin{array}{c}
g x \preceq g y, \\
g y \preceq g x
\end{array}\right\} \Rightarrow\left\{\begin{array}{c}
T x \preceq T y, \\
T y \preceq T x
\end{array}\right\} \Rightarrow T x=T y .
$$


Definition 1.18. [19]. Let $(X, \preceq)$ be a partially ordered set and endow the product space $X^{2}$ with the following partial order:

$$
(u, v) \sqsubseteq(x, y) \Leftrightarrow x \succeq u \text { and } y \preceq v \text {, for all }(u, v),(x, y) \in X^{2} .
$$

Definition 1.19. $[4,11,18,19]$. Let $(X, d, \preceq)$ be a partially ordered metric space. Two mappings $T, g: X \rightarrow X$ are said to be O-compatible if

$$
\lim _{n \rightarrow \infty} d\left(g T x_{n}, \operatorname{Tg} x_{n}\right)=0
$$

provided that $\left\{x_{n}\right\}$ is a sequence in $X$ such that $\left\{g x_{n}\right\}$ is $\preceq$-monotone, that is, it is either non-increasing or non-decreasing with respect to $\preceq$ and

$$
\lim _{n \rightarrow \infty} T x_{n}=\lim _{n \rightarrow \infty} g x_{n} \in X \text {. }
$$

Samet et al. [23] declared that most of the coupled fixed point theorems for single-valued mappings on ordered metric spaces can be derived from well-known fixed point theorems.

On the other hand, Ding et al. [7] proved coupled coincidence and common coupled fixed point theorems for generalized nonlinear contraction on partially ordered metric spaces which generalize the results of Lakshmikantham and Ciric [16]. Our fundamental sources are $[2,5,6,7,8,12,13,14,15,19,20,21,22,23]$.

In this paper, we obtain a coincidence point theorem for $g$-non-decreasing mappings satisfying new contractive condition on partially ordered metric spaces. With the help of our result, we derive a coupled coincidence point theorem of generalized compatible pair of mappings $F, G: X^{2} \rightarrow X$. We also give an example and an application to integral equations to support our results. Our results generalize, extend, modify, improve and sharpen the results of Gnana-Bhaskar and Lakshmikantham [3] and Lakshmikantham and Ciric [16].

\section{Main results}

Lemma 2.1. Let $(X, d)$ be a metric space. Suppose $Y=X^{2}$ and define $\delta$ : $Y \times Y \rightarrow[0,+\infty)$ by

$$
\delta((x, y),(u, v))=\max \{d(x, u), d(y, v)\}, \text { for all }(x, y),(u, v) \in Y .
$$

Then $\delta$ is metric on $Y$ and $(X, d)$ is complete if and only if $(Y, \delta)$ is complete.

Let $\Phi$ denote the set of all functions $\varphi:[0,+\infty) \rightarrow[0,+\infty)$ satisfying

$\left(i_{\varphi}\right) \varphi$ is non-decreasing,

$\left(i i_{\varphi}\right) \varphi(t)<t$ for all $t>0$,

$\left(i i i_{\varphi}\right) \lim _{r \rightarrow t+} \varphi(r)<t$ for all $t>0$. 
Let $\Psi$ denote the set of all functions $\psi:[0,+\infty) \rightarrow[0,+\infty)$ which satisfies $\left(i_{\psi}\right) \psi$ is continuous and non-decreasing, $\left(i i_{\psi}\right) \psi(t)<t$ for all $t>0$.

Note that, by $\left(i_{\psi}\right)$ and $\left(i i_{\psi}\right)$ we have that $\psi(t)=0$ if and only if $t=0$.

Theorem 2.1. Let $(X, d, \preceq)$ be a partially ordered metric space and let $T, g$ : $X \rightarrow X$ be two mappings such that the following properties are fulfilled:

(i) $T(X) \subseteq g(X)$,

(ii) $T$ is $(g, \preceq)$-non-decreasing,

(iii) there exists $x_{0} \in X$ such that $g x_{0} \preceq T x_{0}$,

(iv) there exists $\varphi \in \Phi$ and $\psi \in \Psi$ such that

$$
d(T x, T y) \leq \varphi(d(g x, g y))+\psi(M(x, y)),
$$

where

$$
M(x, y)=\min \{d(g x, T x), d(g y, T y), d(g x, T y), d(g y, T x)\},
$$

for all $x, y \in X$ such that $g x \preceq g y$. Also assume that, at least, one of the following conditions holds:

(a) $(X, d)$ is complete, $T$ and $g$ are continuous and the pair $(T, g)$ is $O$ compatible,

(b) $(X, d)$ is complete, $T$ and $g$ are continuous and commuting,

(c) $(g(X), d)$ is complete and $(X, d, \preceq)$ is non-decreasing-regular,

$(d)(X, d)$ is complete, $g(X)$ is closed and $(X, d, \preceq)$ is non-decreasing-regular,

$(e)(X, d)$ is complete, $g$ is continuous, the pair $(T, g)$ is O-compatible and $(X$, $d, \preceq)$ is non-decreasing-regular.

Then $T$ and $g$ have, at least, a coincidence point.

Proof. We divide the proof into four steps.

Step 1. We claim that there exists a sequence $\left\{x_{n}\right\} \subseteq X$ such that $\left\{g x_{n}\right\}$ is $\preceq$-non-decreasing and $g x_{n+1}=T x_{n}$, for all $n \geq 0$. Let $x_{0} \in X$ be arbitrary. Since $T x_{0} \in T(X) \subseteq g(X)$, therefore there exists $x_{1} \in X$ such that $T x_{0}=g x_{1}$. Then $g x_{0} \preceq T x_{0}=g x_{1}$. Since $T$ is $(g, \preceq)$-non-decreasing, therefore $T x_{0} \preceq T x_{1}$. Again, since $T x_{1} \in T(X) \subseteq g(X)$, therefore there exists $x_{2} \in X$ such that $T x_{1}=g x_{2}$. Then $g x_{1}=T x_{0} \preceq T x_{1}=g x_{2}$. Since $T$ is $(g, \preceq)$-non-decreasing, therefore $T x_{1} \preceq T x_{2}$. Repeating this argument, there exists a sequence $\left\{x_{n}\right\}_{n=0}^{\infty}$ such that $\left\{g x_{n}\right\}$ is $\preceq$ non-decreasing, $g x_{n+1}=T x_{n} \preceq T x_{n+1}=g x_{n+2}$ and

$$
g x_{n+1}=T x_{n} \text { for all } n \geq 0 .
$$

Step 2. We claim that $\left\{g x_{n}\right\}_{n=0}^{\infty}$ is a Cauchy sequence in $X$. Now, by contractive condition $(i v)$, we have

$$
\begin{aligned}
& d\left(g x_{n}, g x_{n+1}\right) \\
= & d\left(T x_{n-1}, T x_{n}\right) \\
\leq & \varphi\left(d\left(g x_{n-1}, g x_{n}\right)\right)+\psi\left(M\left(x_{n-1}, x_{n}\right)\right) .
\end{aligned}
$$


Thus, by the fact that $\psi(0)=0$, we get

$$
d\left(g x_{n}, g x_{n+1}\right) \leq \varphi\left(d\left(g x_{n-1}, g x_{n}\right)\right),
$$

which, by $\left(i i_{\varphi}\right)$, implies

$$
d\left(g x_{n}, g x_{n+1}\right)<d\left(g x_{n-1}, g x_{n}\right) .
$$

This shows that the sequence $\left\{\delta_{n}\right\}_{n=0}^{\infty}$ defined by $\delta_{n}=d\left(g x_{n}, g x_{n+1}\right)$, is a decreasing sequence of positive numbers. Then there exists $\delta \geq 0$ such that

$$
\lim _{n \rightarrow \infty} \delta_{n}=\lim _{n \rightarrow \infty} d\left(g x_{n}, g x_{n+1}\right)=\delta .
$$

We shall prove that $\delta=0$. Suppose that $\delta>0$. Letting $n \rightarrow \infty$ in (2.3), by using (2.4) and $\left(i i_{\varphi}\right)$, we get

$$
\delta \leq \lim _{n \rightarrow \infty} \varphi\left(\delta_{n}\right)=\lim _{\delta_{n} \rightarrow \delta+} \varphi\left(\delta_{n}\right)<\delta,
$$

which is a contradiction. Hence

$$
\delta=\lim _{n \rightarrow \infty} \delta_{n}=\lim _{n \rightarrow \infty} d\left(g x_{n}, g x_{n+1}\right)=0 .
$$

We now prove that $\left\{g x_{n}\right\}_{n=0}^{\infty}$ is a Cauchy sequence in $(X, d)$. Suppose, to the contrary, that it is not a Cauchy sequence. Then there exists an $\varepsilon>0$ for which we can find subsequences $\left\{g x_{n(k)}\right\},\left\{g x_{m(k)}\right\}$ of $\left\{g x_{n}\right\}_{n=0}^{\infty}$ such that

$$
d\left(g x_{n(k)}, g x_{m(k)}\right) \geq \varepsilon, k=1,2, \ldots
$$

We can choose $n(k)$ to be the smallest positive integer satisfying (2.6). So

$$
d\left(g x_{n(k)-1}, g x_{m(k)}\right)<\varepsilon .
$$

By (2.6), (2.7) and the triangle inequality, we have

$$
\begin{aligned}
\varepsilon & \leq r_{k}=d\left(g x_{n(k)}, g x_{m(k)}\right) \\
& \leq d\left(g x_{n(k)}, g x_{n(k)-1}\right)+d\left(g x_{n(k)-1}, g x_{m(k)}\right), \\
& <d\left(g x_{n(k)}, g x_{n(k)-1}\right)+\varepsilon .
\end{aligned}
$$

Letting $k \rightarrow \infty$ in the above inequality and using (2.5), we get

$$
\lim _{k \rightarrow \infty} r_{k}=\lim _{k \rightarrow \infty} d\left(g x_{n(k)}, g x_{m(k)}\right)=\varepsilon .
$$

By the triangle inequality, we have

$$
\begin{aligned}
r_{k}= & d\left(g x_{n(k)}, g x_{m(k)}\right) \\
\leq & d\left(g x_{n(k)}, g x_{n(k)+1}\right)+d\left(g x_{n(k)+1}, g x_{m(k)+1}\right) \\
& +d\left(g x_{m(k)+1}, g x_{m(k)}\right) \\
\leq & \delta_{n(k)}+\delta_{m(k)}+d\left(g x_{n(k)+1}, g x_{m(k)+1}\right) .
\end{aligned}
$$


Thus

$$
r_{k} \leq \delta_{n(k)}+\delta_{m(k)}+d\left(g x_{n(k)+1}, g x_{m(k)+1}\right) .
$$

Now, by contractive condition $(i v)$, we have

$$
\begin{aligned}
& d\left(g x_{n(k)+1}, g x_{m(k)+1}\right) \\
= & d\left(T x_{n(k)}, T x_{m(k)}\right) \\
\leq & \varphi\left(d\left(g x_{n(k)}, g x_{m(k)}\right)\right)+\psi\left(M\left(x_{n(k)}, x_{m(k)}\right)\right) \\
\leq & \varphi\left(r_{k}\right)+\psi\left(M\left(x_{n(k)}, x_{m(k)}\right)\right) .
\end{aligned}
$$

Thus

$$
d\left(g x_{n(k)+1}, g x_{m(k)+1}\right) \leq \varphi\left(r_{k}\right)+\psi\left(M\left(x_{n(k)}, x_{m(k)}\right)\right) .
$$

By (2.9) and (2.10), we get

$$
r_{k} \leq \delta_{n(k)}+\delta_{m(k)}+\varphi\left(r_{k}\right)+\psi\left(M\left(x_{n(k)}, x_{m(k)}\right)\right) .
$$

Letting $k \rightarrow \infty$ in the above inequality, by using (2.5), (2.8), $\left(i i i_{\varphi}\right)$ and by the fact that $\psi(t)<t$ for all $t>0$, we get

$$
\varepsilon \leq 0+0+\lim _{k \rightarrow \infty} \varphi\left(r_{k}\right)+0 \leq \lim _{r_{k} \rightarrow \varepsilon+} \varphi\left(r_{k}\right)<\varepsilon,
$$

which is a contradiction. This shows that $\left\{g x_{n}\right\}_{n=0}^{\infty}$ is a Cauchy sequence in $X$.

Step 3. We claim that $T$ and $g$ have a coincidence point distinguishing between cases $(a)-(e)$.

Suppose now that $(a)$ holds, that is, $(X, d)$ is complete, $T$ and $g$ are continuous and the pair $(T, g)$ is O-compatible. Since $(X, d)$ is complete, therefore there exists $z \in X$ such that $\left\{g x_{n}\right\} \rightarrow z$ and $\left\{T x_{n}\right\} \rightarrow z$. Since $T$ and $g$ are continuous, therefore $\left\{T g x_{n}\right\} \rightarrow T z$ and $\left\{g g x_{n}\right\} \rightarrow g z$. Since the pair $(T, g)$ is O-compatible, therefore $\lim _{n \rightarrow \infty} d\left(g T x_{n}, T g x_{n}\right)=0$. Thus, we conclude that

$$
d(g z, T z)=\lim _{n \rightarrow \infty} d\left(g g x_{n+1}, T g x_{n}\right)=\lim _{n \rightarrow \infty} d\left(g T x_{n}, T g x_{n}\right)=0
$$

that is, $z$ is a coincidence point of $T$ and $g$.

Suppose now that $(b)$ holds, that is, $(X, d)$ is complete, $T$ and $g$ are continuous and commuting. It is evident that $(b)$ implies $(a)$.

Suppose now that $(c)$ holds, that is, $(g(X), d)$ is complete and $(X, d, \preceq)$ is nondecreasing-regular. As $\left\{g x_{n}\right\}$ is a Cauchy sequence in the complete space $(g(X)$, $d)$, so there exists $y \in g(X)$ such that $\left\{g x_{n}\right\} \rightarrow y$. Let $z \in X$ be any point such that $y=g z$, then $\left\{g x_{n}\right\} \rightarrow g z$. Indeed, as $(X, d, \preceq)$ is non-decreasing-regular and $\left\{g x_{n}\right\}$ is $\preceq$-non-decreasing and converging to $g z$, we deduce that $g x_{n} \preceq g z$ for all $n \geq 0$. Applying the contractive condition $(i v)$, we get

$$
d\left(g x_{n+1}, T z\right)=d\left(T x_{n}, T z\right) \leq \varphi\left(d\left(g x_{n}, g z\right)\right)+\psi\left(M\left(x_{n}, z\right)\right),
$$


Thus, by using the continuity of $\psi$ and $\left(i i_{\varphi}\right)$, we get

$$
d(g z, T z) \leq \lim _{t \rightarrow 0+} \varphi(t)+0=0+0=0 .
$$

Hence we must have $d(g z, T z)=0$, that is, $z$ is a coincidence point of $T$ and $g$.

Suppose now that $(d)$ holds, that is, $(X, d)$ is complete, $g(X)$ is closed and $(X$, $d, \preceq)$ is non-decreasing-regular. It follows from the fact that a closed subset of a complete metric space is also complete. Then, $(g(X), d)$ is complete and $(X, d, \preceq)$ is non-decreasing-regular. Thus $(c)$ can apply here.

Suppose now that $(e)$ holds, that is, $(X, d)$ is complete, $g$ is continuous, the pair $(T, g)$ is O-compatible and $(X, d, \preceq)$ is non-decreasing-regular. As $(X, d)$ is complete, so there exists $z \in X$ such that $\left\{g x_{n}\right\} \rightarrow z$. Since $T x_{n}=g x_{n+1}$ for all $n \geq 0$, we also have that $\left\{T x_{n}\right\} \rightarrow z$. As $g$ is continuous, then $\left\{g g x_{n}\right\} \rightarrow g z$. Furthermore, since the pair $(T, g)$ is O-compatible, we have $\lim _{n \rightarrow \infty} d\left(g g x_{n+1}\right.$, $\left.T g x_{n}\right)=\lim _{n \rightarrow \infty} d\left(g T x_{n}, T g x_{n}\right)=0$. As $\left\{g g x_{n}\right\} \rightarrow g z$ the previous property means that $\left\{T g x_{n}\right\} \rightarrow g z$.

Indeed, as $(X, d, \preceq)$ is non-decreasing-regular and $\left\{g x_{n}\right\}$ is $\preceq$-non-decreasing and converging to $z$, we deduce that $g x_{n} \preceq z$ for all $n \geq 0$. Applying the contractive condition $(i v)$, we get

$$
d\left(T g x_{n}, T z\right) \leq \varphi\left(d\left(g g x_{n}, g z\right)\right)+\psi\left(M\left(g x_{n}, z\right)\right) .
$$

Since $\left\{g g x_{n}\right\} \rightarrow g z$, therefore by using the continuity of $\psi$ and $\left(i i i_{\varphi}\right)$, we get

$$
d(g z, T z) \leq \lim _{t \rightarrow 0+} \varphi(t)+0=0+0=0 .
$$

Hence we must have $d(g z, T z)=0$, that is, $z$ is a coincidence point of $T$ and $g$.

Next, we derive the two dimensional version of Theorem 2.1. For the ordered metric space $(X, d, \preceq)$, let us consider the ordered metric space $\left(X^{2}, \delta, \sqsubseteq\right)$, where $\delta$ was defined in Lemma 2.1 and $\sqsubseteq$ was introduced in (1.1). Define the mappings $T_{F}, T_{G}: X^{2} \rightarrow X^{2}$, for all $(x, y) \in X^{2}$, by,

$$
T_{F}(x, y)=(F(x, y), F(y, x)) \text { and } T_{G}(x, y)=(G(x, y), G(y, x)) .
$$

Under these conditions, the following properties hold:

Lemma 2.2. Let $(X, d, \preceq)$ be a partially ordered metric space and let $F, G$ : $X^{2} \rightarrow X$ be two mappings. Then

(1) $(X, d)$ is complete if and only if $\left(X^{2}, \delta\right)$ is complete.

(2) If $(X, d, \preceq)$ is regular, then $\left(X^{2}, \delta\right.$, $)$ is also regular.

(3) If $F$ is d-continuous, then $T_{F}$ is $\delta$-continuous.

(4) If $F$ is $G$-increasing with respect to $\preceq$, then $T_{F}$ is $\left(T_{G}\right.$, $)$-non-decreasing. 
(5) If there exist two elements $x_{0}, y_{0} \in X$ with $G\left(x_{0}, y_{0}\right) \preceq F\left(x_{0}, y_{0}\right)$ and $G\left(y_{0}, x_{0}\right) \succeq F\left(y_{0}, x_{0}\right)$, then there exists a point $\left(x_{0}, y_{0}\right) \in X^{2}$ such that $T_{G}\left(x_{0}\right.$, $\left.y_{0}\right) \sqsubseteq T_{F}\left(x_{0}, y_{0}\right)$.

(6) For any $x, y \in X$, there exist $u, v \in X$ such that $F(x, y)=G(u, v)$ and $F(y, x)=G(v, u)$, then $T_{F}\left(X^{2}\right) \subseteq T_{G}\left(X^{2}\right)$.

(7) If there exists $\varphi \in \Phi$ and $\psi \in \Psi$ such that

(2.12) $d(F(x, y), F(u, v))$

$\leq \varphi(\max \{d(G(x, y), G(u, v)), d(G(y, x), G(v, u))\})+\psi(M(x, y, u, v))$,

where

$$
M(x, y, u, v)=\min \left\{\begin{array}{c}
d(G(x, y), F(x, y)), d(G(u, v), F(u, v)), \\
d(G(x, y), F(u, v)), d(G(u, v), F(x, y)), \\
d(G(y, x), F(y, x)), d(G(v, u), F(v, u)), \\
d(G(y, x), F(v, u)), d(G(v, u), F(y, x))
\end{array}\right\}
$$

for all $x, y, u, v \in X$, where $G(x, y) \preceq G(u, v)$ and $G(y, x) \succeq G(v, u)$, then

$$
\delta\left(T_{F}(x, y), T_{F}(u, v)\right) \leq \varphi\left(\delta\left(T_{G}(x, y), T_{G}(u, v)\right)\right)+\psi\left(M_{\delta}((x, y),(u, v))\right),
$$

where

$$
M_{\delta}((x, y),(u, v))=\min \left\{\begin{array}{l}
\delta\left(T_{G}(x, y), T_{F}(x, y)\right), \\
\delta\left(T_{G}(u, v), T_{F}(u, v)\right), \\
\delta\left(T_{G}(x, y), T_{F}(u, v)\right), \\
\delta\left(T_{G}(u, v), T_{F}(x, y)\right)
\end{array}\right\},
$$

for all $(x, y),(u, v) \in X^{2}$, where $T_{G}(x, y) \sqsubseteq T_{G}(u, v)$.

(8) If the pair $\{F, G\}$ is generalized compatible, then the mappings $T_{F}$ and $T_{G}$ are $O$-compatible in $\left(X^{2}, \delta, \sqsubseteq\right)$.

(9) A point $(x, y) \in X^{2}$ is a coupled coincidence point of $F$ and $G$ if and only if it is a coincidence point of $T_{F}$ and $T_{G}$.

Proof. Statement (1) follows from Lemma 21 and (2), (3), (5), (6) and (9) are obvious.

(4) Assume that $F$ is $G$-increasing with respect to $\preceq$ and let $(x, y),(u, v) \in X^{2}$ be such that $T_{G}(x, y) \sqsubseteq T_{G}(u, v)$. Then $G(x, y) \preceq G(u, v)$ and $G(y, x) \succeq G(v$, $u)$. Since $F$ is $G$-increasing with respect to $\preceq$, we have that $F(x, y) \preceq F(u, v)$ and $F(y, x) \succeq F(v, u)$. Therefore $T_{F}(x, y) \sqsubseteq T_{F}(u, v)$ which shows that $T_{F}$ is $\left(T_{G}\right.$, $\sqsubseteq)$-non-decreasing.

(7) Suppose that there exist $\varphi \in \Phi$ and $\psi \in \Psi$ such that

$$
\begin{aligned}
& d(F(x, y), F(u, v)) \\
\leq & \varphi\left(\max \left\{\begin{array}{c}
d(G(x, y), G(u, v)), \\
d(G(y, x), G(v, u))
\end{array}\right\}\right)+\psi(M(x, y, u, v)),
\end{aligned}
$$


for all $x, y, u, v \in X$, where $G(x, y) \preceq G(u, v)$ and $G(y, x) \succeq G(v, u)$ and let (x, $y),(u, v) \in X^{2}$ be such that $T_{G}(x, y) \sqsubseteq T_{G}(u, v)$. Therefore $G(x, y) \preceq G(u, v)$ and $G(y, x) \succeq G(v, u)$. From (2.12), we have

$$
\begin{aligned}
& d(F(x, y), F(u, v)) \\
\leq & \varphi(\max \{d(G(x, y), G(u, v)), d(G(y, x), G(v, u))\}) \\
+ & \psi(M(x, y, u, v)) .
\end{aligned}
$$

Furthermore $G(y, x) \succeq G(v, u)$ and $G(x, y) \preceq G(u, v)$, the contractive condition (2.12) implies that

$$
\begin{aligned}
& d(F(y, x), F(v, u)) \\
\leq & \varphi(\max \{d(G(x, y), G(u, v)), d(G(y, x), G(v, u))\}) \\
& +\psi(M(x, y, u, v)) .
\end{aligned}
$$

Combining (2.13) and (2.14), we get

$$
\begin{aligned}
& \max \{d(F(x, y), F(u, v)), d(F(y, x), F(v, u))\} \\
\leq & \varphi(\max \{d(G(x, y), G(u, v)), d(G(y, x), G(v, u))\}) \\
& +\psi(M(x, y, u, v)) .
\end{aligned}
$$

It follows from (2.15) that

$$
\begin{aligned}
& \delta\left(T_{F}(x, y), T_{F}(u, v)\right) \\
= & \delta((F(x, y), F(y, x)),(F(u, v), F(v, u))) \\
= & \max \{d(F(x, y), F(u, v)), d(F(y, x), F(v, u))\} \\
\leq & \varphi(\max \{d(G(x, y), G(u, v)), d(G(y, x), G(v, u))\}) \\
& +\psi(M(x, y, u, v)) \\
\leq & \varphi(\delta((G(x, y), G(y, x)),(G(u, v), G(v, u)))) \\
& +\psi\left(M_{\delta}((x, y),(u, v))\right) \\
\leq & \varphi\left(\delta\left(T_{G}(x, y), T_{G}(u, v)\right)\right)+\psi\left(M_{\delta}((x, y),(u, v))\right) .
\end{aligned}
$$

(8) Let $\left\{\left(x_{n}, y_{n}\right)\right\} \subseteq X^{2}$ be any sequence such that $T_{F}\left(x_{n}, y_{n}\right) \stackrel{\delta}{\rightarrow}(x, y)$ and $T_{G}\left(x_{n}, y_{n}\right) \stackrel{\delta}{\rightarrow}(x, y)$ (Note that it is not require to suppose that $\left\{T_{G}\left(x_{n}, y_{n}\right)\right\}$ is $\sqsubseteq$-monotone). Thus

$$
\begin{aligned}
& \left(F\left(x_{n}, y_{n}\right), F\left(y_{n}, x_{n}\right)\right) \stackrel{\delta}{\rightarrow}(x, y) \\
\Rightarrow \quad & F\left(x_{n}, y_{n}\right) \stackrel{d}{\rightarrow} x \text { and } F\left(y_{n}, x_{n}\right) \stackrel{d}{\rightarrow} y,
\end{aligned}
$$

and

$$
\begin{aligned}
& \left(G\left(x_{n}, y_{n}\right), G\left(y_{n}, x_{n}\right)\right) \stackrel{\delta}{\rightarrow}(x, y) \\
\Rightarrow \quad & G\left(x_{n}, y_{n}\right) \stackrel{d}{\rightarrow} x \text { and } G\left(y_{n}, x_{n}\right) \stackrel{d}{\rightarrow} y .
\end{aligned}
$$


Therefore

$$
\begin{aligned}
\lim _{n \rightarrow \infty} F\left(x_{n}, y_{n}\right) & =\lim _{n \rightarrow \infty} G\left(x_{n}, y_{n}\right)=x \in X \\
\lim _{n \rightarrow \infty} F\left(y_{n}, x_{n}\right) & =\lim _{n \rightarrow \infty} G\left(y_{n}, x_{n}\right)=y \in X .
\end{aligned}
$$

Since the pair $\{F, G\}$ is generalized compatible, therefore

$$
\begin{aligned}
& \lim _{n \rightarrow \infty} d\left(F\left(G\left(x_{n}, y_{n}\right), G\left(y_{n}, x_{n}\right)\right), G\left(F\left(x_{n}, y_{n}\right), F\left(y_{n}, x_{n}\right)\right)\right)=0, \\
& \lim _{n \rightarrow \infty} d\left(F\left(G\left(y_{n}, x_{n}\right), G\left(x_{n}, y_{n}\right)\right), G\left(F\left(y_{n}, x_{n}\right), F\left(x_{n}, y_{n}\right)\right)\right)=0 .
\end{aligned}
$$

In particular,

$$
\begin{aligned}
& \lim _{n \rightarrow \infty} \delta\left(T_{G} T_{F}\left(x_{n}, y_{n}\right), T_{F} T_{G}\left(x_{n}, y_{n}\right)\right) \\
= & \lim _{n \rightarrow \infty} \delta\left(T_{G}\left(F\left(x_{n}, y_{n}\right), F\left(y_{n}, x_{n}\right)\right), T_{F}\left(G\left(x_{n}, y_{n}\right), G\left(y_{n}, x_{n}\right)\right)\right) \\
= & \lim _{n \rightarrow \infty} \delta\left(\begin{array}{c}
\left(G\left(F\left(x_{n}, y_{n}\right), F\left(y_{n}, x_{n}\right)\right), G\left(F\left(y_{n}, x_{n}\right), F\left(x_{n}, y_{n}\right)\right)\right), \\
\left(F\left(G\left(x_{n}, y_{n}\right), G\left(y_{n}, x_{n}\right)\right), F\left(G\left(y_{n}, x_{n}\right), G\left(x_{n}, y_{n}\right)\right)\right)
\end{array}\right) \\
= & \lim _{n \rightarrow \infty} \max \left\{\begin{array}{l}
d\left(G\left(F\left(x_{n}, y_{n}\right), F\left(y_{n}, x_{n}\right)\right), F\left(G\left(x_{n}, y_{n}\right), G\left(y_{n}, x_{n}\right)\right)\right), \\
d\left(G\left(F\left(y_{n}, x_{n}\right), F\left(x_{n}, y_{n}\right)\right), F\left(G\left(y_{n}, x_{n}\right), G\left(x_{n}, y_{n}\right)\right)\right)
\end{array}\right\} \\
= & 0 .
\end{aligned}
$$

Hence, the mappings $T_{F}$ and $T_{G}$ are O-compatible in $\left(X^{2}, \delta, \sqsubseteq\right)$.

Theorem 2.2. Let $(X, \preceq)$ be a partially ordered set such that there exists a complete metric d on $X$. Assume $F, G: X^{2} \rightarrow X$ be two generalized compatible mappings such that $F$ is $G$-increasing with respect to $\preceq, G$ is continuous and there exist two elements $x_{0}, y_{0} \in X$ with

$$
G\left(x_{0}, y_{0}\right) \preceq F\left(x_{0}, y_{0}\right) \text { and } G\left(y_{0}, x_{0}\right) \succeq F\left(y_{0}, x_{0}\right) \text {. }
$$

Suppose that there exist $\varphi \in \Phi$ and $\psi \in \Psi$ satisfying (2.12) and for any $x, y \in X$, there exist $u, v \in X$ such that

$$
F(x, y)=G(u, v) \text { and } F(y, x)=G(v, u)
$$

Also suppose that either

(a) $F$ is continuous or

(b) $(X, d, \preceq)$ is regular.

Then $F$ and $G$ have a coupled coincidence point.

Proof. It is only required to use Theorem 2.1 to the mappings $T=T_{F}$ and $g=T_{G}$ in the ordered metric space $\left(X^{2}, \delta\right.$, $)$ by applying Lemma 2.2. 
Corollary 2.1. Let $(X, \preceq)$ be a partially ordered set such that there exists a complete metric d on $X$. Assume $F, G: X^{2} \rightarrow X$ be two commuting mappings satisfying (2.12) and (2.16) such that $F$ is $G$-increasing with respect to $\preceq, G$ is continuous and there exist two elements $x_{0}, y_{0} \in X$ with

$$
G\left(x_{0}, y_{0}\right) \preceq F\left(x_{0}, y_{0}\right) \text { and } G\left(y_{0}, x_{0}\right) \succeq F\left(y_{0}, x_{0}\right) .
$$

Also suppose that either

(a) $F$ is continuous or

(b) $(X, d, \preceq)$ is regular.

Then $F$ and $G$ have a coupled coincidence point.

Next, we deduce results without the mixed $g$-monotone property of $F$.

Corollary 2.2. Let $(X, \preceq)$ be a partially ordered set such that there exists a complete metric $d$ on $X$. Assume $F: X^{2} \rightarrow X$ and $g: X \rightarrow X$ be two mappings such that $F$ is $g$-increasing with respect to $\preceq$ and there exist $\varphi \in \Phi$ and $\psi \in \Psi$ such that

$$
\begin{aligned}
& d(F(x, y), F(u, v)) \\
\leq & \varphi(\max \{d(g x, g u), d(g y, g v\}))+\psi\left(M_{g}(x, y, u, v)\right),
\end{aligned}
$$

where

$$
M_{g}(x, y, u, v)=\min \left\{\begin{array}{c}
d(g x, F(x, y)), d(g u, F(u, v)), \\
d(g x, F(u, v)), d(g u, F(x, y)), \\
d(g y, F(y, x)), d(g v, F(v, u)), \\
d(g y, F(v, u)), d(g v, F(y, x))
\end{array}\right\}
$$

for all $x, y, u, v \in X$, where $g x \preceq g u$ and $g y \succeq g v$. Suppose that $F(X \times X) \subseteq g(X)$, $g$ is continuous and the pair $\{F, g\}$ is compatible. Also suppose that either

(a) $F$ is continuous or

(b) $(X, d, \preceq)$ is regular.

If there exist two elements $x_{0}, y_{0} \in X$ with

$$
g x_{0} \preceq F\left(x_{0}, y_{0}\right) \text { and } g y_{0} \succeq F\left(y_{0}, x_{0}\right) \text {. }
$$

Then $F$ and $g$ have a coupled coincidence point.

Corollary 2.3. Let $(X, \preceq)$ be a partially ordered set such that there exists a complete metric $d$ on $X$. Assume $F: X^{2} \rightarrow X$ and $g: X \rightarrow X$ be two mappings satisfying (2.17) such that $F$ is g-increasing with respect to $\preceq$. Suppose that $F(X \times X) \subseteq g(X), g$ is continuous and the pair $\{F, g\}$ is commuting. Also suppose that either

(a) $F$ is continuous or

(b) $(X, d, \preceq)$ is regular. 
If there exist two elements $x_{0}, y_{0} \in X$ with

$$
g x_{0} \preceq F\left(x_{0}, y_{0}\right) \text { and } g y_{0} \succeq F\left(y_{0}, x_{0}\right) \text {. }
$$

Then $F$ and $g$ have a coupled coincidence point.

Now, we deduce the result without the mixed monotone property of $F$.

Corollary 2.4. Let $(X, \preceq)$ be a partially ordered set such that there exists a complete metric $d$ on $X$. Assume $F: X^{2} \rightarrow X$ be an increasing mapping with respect to $\preceq$ and there exist $\varphi \in \Phi$ and $\psi \in \Psi$ such that

(2.18) $d(F(x, y), F(u, v)) \leq \varphi(\max \{d(x, u), d(y, v)\}))+\psi(m(x, y, u, v))$,

where

$$
m(x, y, u, v)=\min \left\{\begin{array}{l}
d(x, F(x, y)), d(u, F(u, v)), \\
d(x, F(u, v)), d(u, F(x, y)), \\
d(y, F(y, x)), d(v, F(v, u)), \\
d(y, F(v, u)), d(v, F(y, x))
\end{array}\right\}
$$

for all $x, y, u, v \in X$, where $x \preceq u$ and $y \succeq v$. Also suppose that either

(a) $F$ is continuous or

(b) $(X, d, \preceq)$ is regular.

If there exist two elements $x_{0}, y_{0} \in X$ with

$$
x_{0} \preceq F\left(x_{0}, y_{0}\right) \text { and } y_{0} \succeq F\left(y_{0}, x_{0}\right) \text {. }
$$

Then $F$ has a coupled fixed point.

Example 2.1. Suppose that $X=[0,1]$, equipped with the usual metric $d: X^{2} \rightarrow[0$, $+\infty)$ with the natural ordering of real numbers $\leq$. Let $F, G: X^{2} \rightarrow X$ be defined as

$$
\begin{aligned}
& F(x, y)=\left\{\begin{array}{c}
\frac{x^{2}-y^{2}}{3}, \text { if } x \geq y, \\
0, \text { if } x<y,
\end{array}\right. \\
& G(x, y)=\left\{\begin{array}{c}
x^{2}-y^{2}, \text { if } x \geq y, \\
0, \text { if } x<y .
\end{array}\right.
\end{aligned}
$$

Define $\varphi:[0,+\infty) \rightarrow[0,+\infty)$ by

$$
\varphi(t)=\left\{\begin{array}{l}
\frac{t}{3}, \text { for } t \neq 1 \\
\frac{3}{4}, \text { for } t=1
\end{array}\right.
$$

and $\psi:[0,+\infty) \rightarrow[0,+\infty)$ by

$$
\psi(t)=\frac{t}{4}, \text { for all } t \geq 0 .
$$


First, we shall show that the contractive condition (2.12) holds for the mappings $F$ and $G$. Let $x, y, u, v \in X$ such that $G(x, y) \preceq G(u, v)$ and $G(y, x) \succeq G(v, u)$, we have

$$
\begin{aligned}
& d(F(x, y), F(u, v)) \\
= & \left|\frac{x^{2}-y^{2}}{3}-\frac{u^{2}-v^{2}}{3}\right| \\
= & \frac{1}{3}|G(x, y)-G(u, v)| \\
= & \frac{1}{3} d(G(x, y), G(u, v)) \\
\leq & \frac{1}{3} \max \{d(G(x, y), G(u, v)), d(G(y, x), G(v, u))\} \\
\leq & \varphi(\max \{d(G(x, y), G(u, v)), d(G(y, x), G(v, u))\}) \\
& +\psi(M(x, y, u, v)) .
\end{aligned}
$$

Thus the contractive condition (2.12) holds for all $x, y, u, v \in X$. In addition, like in [12], all the other conditions of Theorem 2.2 are satisfied and $z=(0,0)$ is a coupled coincidence point of $F$ and $G$.

\section{Application to integral equations}

We study the existence of the solution to a nonlinear Fredholm nonlinear integral equation, as an application of the results established in the previous section. We shall consider the following integral equation for all $p \in I=[a, b]$.

$$
x(p)=\int_{a}^{b}\left(K_{1}(p, q)+K_{2}(p, q)\right)[f(q, x(q))+g(q, x(q))] d q+h(p) .
$$

Let $\Theta$ denote the set of all functions $\theta:[0,+\infty) \rightarrow[0,+\infty)$ satisfying

$\left(i_{\theta}\right) \theta$ is non-decreasing,

$\left(i i_{\theta}\right) \theta(p) \leq \frac{1}{3} p$.

Condition 3.1. We assume that the functions $K_{1}, K_{2}, f, g$ fulfill the following conditions:

(i) $K_{1}(p, q) \geq 0$ and $K_{2}(p, q) \leq 0$ for all $p, q \in I$.

(ii) There exist positive numbers $\lambda, \mu$ and $\theta \in \Theta$ such that for all $x, y \in \mathbb{R}$ with $x \succeq y$, the following conditions hold:

$$
\begin{aligned}
0 & \leq f(q, x)-f(q, y) \leq \lambda \theta(x-y), \\
-\mu \theta(x-y) & \leq g(q, x)-g(q, y) \leq 0 .
\end{aligned}
$$

$$
\max \{\lambda, \mu\} \sup _{p \in I} \int_{a}^{b}\left[K_{1}(p, q)-K_{2}(p, q)\right] d q \leq \frac{1}{2}
$$


Definition 3.1. [17]. A pair $(\alpha, \beta) \in X^{2}$ with $X=C(I, \mathbb{R})$, where $C(I, \mathbb{R})$ denote the set of all continuous functions from $I$ to $\mathbb{R}$, is called a coupled lowerupper solution of (3.1) if, for all $p \in I$,

$$
\begin{aligned}
\alpha(p) \leq & \int_{a}^{b} K_{1}(p, q)[f(q, \alpha(q))+g(q, \beta(q))] d q \\
& +\int_{a}^{b} K_{2}(p, q)[f(q, \beta(q))+g(q, \alpha(q))] d q+h(p), \\
\beta(p) \geq & \int_{a}^{b} K_{1}(p, q)[f(q, \beta(q))+g(q, \alpha(q))] d q \\
& +\int_{a}^{b} K_{2}(p, q)[f(q, \alpha(q))+g(q, \beta(q))] d q+h(p) .
\end{aligned}
$$

Theorem 3.1. Consider the integral equation (3.1) with $K_{1}, K_{2} \in C(I \times I, \mathbb{R}), f$, $g \in C(I \times \mathbb{R}, \mathbb{R})$ and $h \in C(I, \mathbb{R})$. Suppose that there exists a coupled lower-upper solution $(\alpha, \beta)$ of (3.1) and Condition 3.1 is satisfied. Then the integral equation (3.1) has a solution in $C(I, \mathbb{R})$.

Proof. Consider $X=C(I, \mathbb{R})$, the natural partial order relation, that is, for $x$, $y \in C(I, \mathbb{R})$, we have

$$
x \preceq y \Longleftrightarrow x(p) \leq y(p), \forall p \in I .
$$

It is clear that $X$ is a complete metric space with respect to the sup metric

$$
d(x, y)=\sup _{p \in I}|x(p)-y(p)| .
$$

Consider the following partial order on $X \times X$ : for $(x, y),(u, v) \in X \times X$,

$$
(x, y) \preceq(u, v) \Longleftrightarrow x(p) \leq u(p) \text { and } y(p) \geq v(p) \text {, for all } p \in I \text {. }
$$

Define $\varphi:[0,+\infty) \rightarrow[0,+\infty)$ by

$$
\varphi(t)=\left\{\begin{array}{l}
\frac{t}{3}, \text { for } t \neq 1, \\
\frac{3}{4}, \text { for } t=1,
\end{array}\right.
$$

and define $\psi:[0,+\infty) \rightarrow[0,+\infty)$ by

$$
\psi(t)=\frac{t}{4}, \text { for all } t \geq 0 .
$$


and the mapping $F: X \times X \rightarrow X$ by

$$
\begin{aligned}
F(x, y)(p)= & \int_{a}^{b} K_{1}(p, q)[f(q, x(q))+g(q, y(q))] d q \\
& +\int_{a}^{b} K_{2}(p, q)[f(q, y(q))+g(q, x(q))] d q+h(p),
\end{aligned}
$$

for all $p \in I$. It is easy to prove, like in [12], that $F$ is increasing. Now for $x, y, u$, $v \in X$ with $x \succeq u$ and $y \preceq v$, we have

$$
\begin{aligned}
& F(x, y)(p)-F(u, v)(p) \\
= & \int_{a}^{b} K_{1}(p, q)[(f(q, x(q))-f(q, u(q)))-(g(q, v(q))-g(q, y(q)))] d q \\
& -\int_{a}^{b} K_{2}(p, q)[(f(q, v(q))-f(q, y(q)))-(g(q, x(q))-g(q, u(q)))] d q .
\end{aligned}
$$

Thus, by using (3.2) and (3.3), we get

$$
\begin{aligned}
& F(x, y)(p)-F(u, v)(p) \\
\leq & \int_{a}^{b} K_{1}(p, q)[\lambda \theta(x(q)-u(q))+\mu \theta(v(q)-y(q))] d q \\
& -\int_{a}^{b} K_{2}(p, q)[\lambda \theta(v(q)-y(q))+\mu \theta(x(q)-u(q))] d q .
\end{aligned}
$$

Since $\theta$ is non-decreasing and $x \succeq u$ and $y \preceq v$, we have

$$
\begin{aligned}
& \theta(x(q)-u(q)) \leq \theta\left(\sup _{q \in I}|x(q)-u(q)|\right)=\theta(d(x, u)) \\
& \theta(v(q)-y(q)) \leq \theta\left(\sup _{q \in I}|v(q)-y(q)|\right)=\theta(d(y, v)) .
\end{aligned}
$$


Hence by $(3.5)$, in fact that $K_{2}(p, q) \leq 0$, we obtain

$$
\begin{aligned}
& |F(x, y)(p)-F(u, v)(p)| \\
\leq & \int_{a}^{b} K_{1}(p, q)[\lambda \theta(d(x, u))+\mu \theta(d(y, v))] d q \\
& -\int_{a}^{b} K_{2}(p, q)[\lambda \theta(d(y, v))+\mu \theta(d(x, u))] d q \\
\leq & \int_{a}^{b} K_{1}(p, q)[\max \{\lambda, \mu\} \theta(d(x, u))+\max \{\lambda, \mu\} \theta(d(y, v))] d q \\
- & \int_{a}^{b} K_{2}(p, q)[\max \{\lambda, \mu\} \theta(d(y, v))+\max \{\lambda, \mu\} \theta(d(x, u))] d q .
\end{aligned}
$$

Since the objects on the right hand side of (3.5) are non-negative. Taking the supremum with respect to $p$, by using (3.4), we get

$$
\begin{aligned}
& d(F(x, y), F(u, v)) \\
\leq & \max \{\lambda, \mu\} \sup _{p \in I} \int_{a}^{b}\left(K_{1}(p, q)-K_{2}(p, q)\right) d q \cdot[\theta(d(x, u))+\theta(d(y, v))] \\
\leq & \frac{\theta(d(x, u))+\theta(d(y, v))}{2} .
\end{aligned}
$$

Thus

$$
d(F(x, y), F(u, v)) \leq \frac{\theta(d(x, u))+\theta(d(y, v))}{2}
$$

Now, since $\theta$ is non-decreasing, we have

$$
\begin{aligned}
\theta(d(x, u)) & \leq \theta(\max \{d(x, u), d(y, v)\}) \\
\theta(d(y, v)) & \leq \theta(\max \{d(x, u), d(y, v)\})
\end{aligned}
$$

which implies, by $\left(i i_{\theta}\right)$, that

$$
\begin{aligned}
\frac{\theta(d(x, u))+\theta(d(y, v))}{2} & \leq \theta(\max \{d(x, u), d(y, v)\}) \\
& \leq \frac{1}{3} \max \{d(x, u), d(y, v)\}
\end{aligned}
$$


Thus by (3.6) and (3.7), we have

$$
\begin{aligned}
& d(F(x, y), F(u, v)) \\
\leq & \frac{1}{3} \max \{d(x, u), d(y, v)\} \\
\leq & \varphi(\max \{d(x, u), d(y, v)\}) \\
\leq & \varphi(\max \{d(x, u), d(y, v)\})+\psi(m(x, y, u, v))
\end{aligned}
$$

which is the contractive condition of Corollary 2.4. Now, let $(\alpha, \beta) \in X \times X$ be a coupled upper-lower solution of (3.1), then we have $\alpha(p) \leq F(\alpha, \beta)(p)$ and $\beta(p) \geq F(\beta, \alpha)(p)$, for all $p \in I$, which shows that all hypothesis of Corollary 2.4 are satisfied. This proves that $F$ has a coupled fixed point $(x, y) \in X \times X$ which is the solution in $X=C(I, \mathbb{R})$ of the integral equation (3.1).

Remark 3.1. Using the same technique that can be used in $[13-15,19,22,23]$ it is possible to derive tripled, quadruple and, in general, multidimensional coincidence point theorems from Theorem 2.1.

\section{R E F E R E N C E S}

1. R.P. Agarwal, R.K. Bisht AND N. ShahZAD: A comparison of various noncommuting conditions in metric fixed point theory and their applications. Fixed Point Theory Appl. 2014, Article ID 38.

2. A. Alotaibi, M. Mursaleen and S.A. Mohiuddine: Some fixed point theorems for Meir-Keeler condensing operators with applications to integral equations. Bull. Belg. Math. Soc. Simon Stevin 22 (2015), 529-541.

3. T.G. Bhaskar and V. Lakshmikantham: Fixed point theorems in partially ordered metric spaces and applications. Nonlinear Anal. 65 (7) (2006), 1379-1393.

4. B.S. ChOudhury AND A. Kundu: A coupled coincidence point results in partially ordered metric spaces for compatible mappings. Nonlinear Anal. 73 (2010), 2524-2531.

5. B. DEShPANDE AND A. HANDA: Nonlinear mixed monotone-generalized contractions on partially ordered modified intuitionistic fuzzy metric spaces with application to integral equations. Afr. Mat. 26 (3-4) (2015), 317-343.

6. B. Deshpande And A. Handa: Application of coupled fixed point technique in solving integral equations on modified intuitionistic fuzzy metric spaces. Adv. Fuzzy Syst. Volume 2014, Article ID 348069.

7. H.S. Ding, L. LI AND S. RADENOVIC: Coupled coincidence point theorems for generalized nonlinear contraction in partially ordered metric spaces. Fixed Point Theory Appl. 2012, 96.

8. I.M. Erhan, E. Karapinar, A. Roldan And N. Shahzad: Remarks on coupled coincidence point results for a generalized compatible pair with applications. Fixed Point Theory Appl. 2014, 207.

9. K. Goebel: A coincidence theorem. Bull. Acad. Pol. Sci., Ser. Sci. Math. Astron. Phys. 16 (1968), 733-735. 
10. D. Guo And V. Lakshmikantham: Coupled fixed points of nonlinear operators with applications. Nonlinear Anal. 11 (5) (1987), 623-632.

11. N.M. Hung, E. Karapinar And N.V. Luong: Coupled coincidence point theorem for O-compatible mappings via implicit relation. Abstr. Appl. Anal. 2012, Article ID 796964 .

12. N. Hussain, M. Abbas, A. Azam and J. Ahmad: Coupled coincidence point results for a generalized compatible pair with applications. Fixed Point Theory Appl. 2014, 62.

13. E. Karapinar And A. Roldan: A note on n-Tuplet fixed point theorems for contractive type mappings in partially ordered metric spaces. J. Inequal. Appl. 2013, Article ID 567.

14. E. Karapinar, A. Roldan, C. Roldan And J. Martinez-Moreno: A note on $N$-Fixed point theorems for nonlinear contractions in partially ordered metric spaces. Fixed Point Theory Appl. 2013, Article ID 310.

15. E. Karapinar, A. Roldan, N. Shahzad And W. Sintunavarat: Discussion on coupled and tripled coincidence point theorems for $\phi$-contractive mappings without the mixed g-monotone property. Fixed Point Theory Appl. 2014, Article ID 92.

16. V. LAKShmikantham AND L. CiRIC: Coupled fixed point theorems for nonlinear contractions in partially ordered metric spaces. Nonlinear Anal. 70 (12) (2009), 4341-4349.

17. N.V. LUONG AND N.X. ThUAN: Coupled fixed points in partially ordered metric spaces and application. Nonlinear Anal. 74 (2011), 983-992.

18. N.V. LUONG AND N.X. ThuAN: Coupled points in ordered generalized metric spaces and application to integro-differential equations. Comput. Math. Appl. 62 (11) (2011), 4238-4248.

19. S.A. Al-Mezel, H. Alsulami, E. Karapinar and A. Roldan: Discussion on multidimensional coincidence points via recent publications. Abstr. Appl. Anal. Volume 2014, Article ID 287492.

20. M. Mursaleen, S. A. Mohiuddine and R. P. Agarwal: Coupled fixed point theorems for $\alpha-\psi$-contractive type mappings in partially ordered metric spaces. Fixed Point Theory Appl. 2012, 228.

21. S. A. Mohiuddine And A. Alotaibi: Some results on a tripled fixed point for nonlinear contractions in partially ordered G-metric spaces. Fixed Point Theory Appl. 2012, 179.

22. A. Roldan, J. Martinez-Moreno, C. Roldan And E. Karapinar: Some remarks on multidimensional fixed point theorems. Fixed Point Theory Appl. 2013, Article ID 158.

23. B. Samet, E. Karapinar, H. Aydi And V.C. Rajic: Discussion on some coupled fixed point theorems. Fixed Point Theory Appl. 2013, 50.

Bhavana Deshpande

Department of Mathematics

Govt. B.S. P.G. College, Jaora

Dist. Ratlam (M.P.), India

E-mail:bhavnadeshpande@yahoo.com 
Amrish Handa

Department of Mathematics

Govt. P. G. Arts and Science College

Ratlam (M.P.), India

E-mail:amrishhanda83@gmail.com 